\title{
RESOURCE-EFFICIENT Wireless ReLAYING Protocols
}

\author{
KYUNGCHUN LeE, SAMSUNG \\ LAJOS HANZO, UNIVERSITY OF SOUTHAMPTON
}

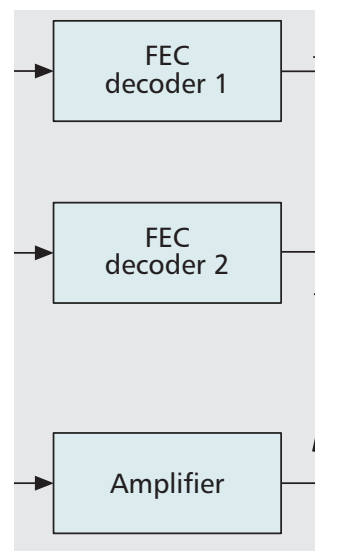

The authors review

various approaches

to creating relay-

aided systems.

The advantages and

disadvantages of

various relaying

schemes are

compared in terms

of slot-efficiency,

error ratio

performance, and

feasibility.

\section{ABSTRACT}

Relay-aided communication is considered one of the key techniques to achieve high throughput at low cost in future wireless systems. However, when transmitting signals via a relay, additional time slots, antennas, or frequency slots are required, which may erode the potential gain of relay-aided systems. In this article various approaches to creating relay-aided systems are reviewed. The advantages and disadvantages of various relaying schemes are compared in terms of their slot efficiency, error rate performance, and feasibility. Our detailed comparisons and the numerical results indicate that the specific family of network coding aided relaying protocols constitutes one of the most promising solutions. We conclude this article by listing a number of open problems.

\section{INTRODUCTION}

Wireless networks are expected to maintain both robust connectivity as well as high data rates in the face of path loss, shadow fading, and intercell interference Dense allocation of full-fledged base stations (BSs) is a feasible solution, but imposes a high infrastructure cost. As another approach to tackle this problem, relay-aided cooperative communication techniques have recently attracted substantial research interest $[1,2]$.

In relay station (RS) aided communications, the mobile station (MS) communicates with the BS assisted by a single or multiple relays, which may be expected to provide better link quality with the BS than direct single-link-based communication. Therefore, relay-aided systems are capable of increasing the attainable data rate, especially in the cell edge region, where MSs typically suffer from both low-power reception and severe intercell interference. Shadow fading can also be mitigated by relays.

Since the provision of a fiber-based RS backhaul is costly, it is preferable to employ RSs that

This work was supported by the Korea Research Foundation Grant funded by the Korean Government (MOEHRD) (KRF2007-357-D00110). The financial support of the EPSRC, UK, and of the EU is also gratefully acknowledged. communicate wirelessly with the BS, especially in rural areas. Wireless RSs also offer high flexibility in terms of their geographic position. For example, when the teletraffic rapidly increases in a certain area, wireless RSs can be installed immediately to provide high-speed communications. The MSs currently not engaged in active communication with the BS may also act as relays without any additional deployment cost.

Although wireless RSs are capable of improving the attainable link quality, again, they require additional radio resources. More explicitly, as illustrated in Fig. 1a, the traditional relaying concept requires four phases to transmit a pair of downlink (DL) and uplink (UL) packets, which is twice as many as direct communications operating without a relay node. This additional radio resource allocation halves the effective throughput and hence erodes the advantages of relaying.

In this article a comparative study of various efficient relaying schemes is presented. More specifically, the recently proposed three-phase and two-phase relaying protocols of Fig. 1b and $1 \mathrm{c}$ are reviewed and compared to the traditional four-phase protocol.

\section{Traditional RELAYING}

As shown in Fig. 1a, traditional relaying schemes require four communication phases. During the first phase, the BS transmits the DL signal to the RS. During the second phase, the RS forwards the received DL signal to the MS. The UL signal is also sent from the MS to the BS during the remaining two communication phases in a twophase reverse-direction manner. Here, the relay's operation can be classified into two types, namely the amplify-and-forward (AF) and decodeand-forward (DF) relaying protocols.

In AF relaying, the relay simply retransmits a scaled version of the received signal without performing any detection or decoding. The simple operation of AF relaying leads to a low-cost lowcomplexity implementation. However, the desired signal and additive noise are jointly amplified at the relay; hence, the AF-RS fails to improve the signal-to-noise ratio (SNR). By contrast, in DF relaying, the relay fully decodes the received signal and hence may succeed in perfectly regenerating the transmitted signal before it forwards the re-encoded packet to the destina- 
tion, provided that the SNR is sufficiently high. Naturally, this is achieved at higher complexity than that of AF relaying.

The destination nodes (the BS in the UL and the MS in the DL) make use of the relayed signal to decode the information bits. For the sake of improving the attainable decoding performance, the signal directly received from the source node can also be utilized. By combining the relayed signal and the direct signal at the destination node, an improved diversity gain can be achieved, because the relay and direct links are typically mutually uncorrelated.

Although the traditional relaying techniques provide a high diversity gain as well as improved link quality, again, they require four communication phases. In a simplistic but appealingly plausible interpretation we may argue that second-order spatial/time diversity may be achieved at the cost of halving the effective throughput. This effect is reminiscent of using second-order time diversity employing $1 / 2$-rate channel coding or second-order frequency diversity invoking two independently faded subcarriers, for example. There are, however, further potential benefits, because we may argue that both the BS-RS and RS-MS links are expected to be of higher quality due to the presence of a line-of-sight (LOS) link. Hence, by exploiting high-order high-throughput modulation and high-rate forward error correction (FEC) codes, an improved bandwidth efficiency may be achievable in both the BS-RS and RS-MS phase.

\section{SUCCESSIVE RELAYING}

Before elaborating on how to reduce the number of time slots required with the aid of Fig. $1 \mathrm{~b}$ and $1 \mathrm{c}$, let us consider a relaying scheme benefiting for an extra RS, which is the successive relaying technique of $[3,4]$, where two parallel RSs are assumed, rather than having a single RS or serially combined multiple RSs. Figure 2 illustrates the operation of successive relaying in the DL. In the first phase seen in Fig. 2a, the BS transmits its DL signal $\mathbf{c}_{1}$ and RS1 listens. In the second phase portrayed in Fig. 2b, RS1 decodes the BS's DL signal $\mathbf{c}_{1}$ received in phase 1 and forwards the reencoded DL packet $\mathbf{c}_{1}$ to the MS, while RS2 receives the DL signal $\mathbf{c}_{2}$ from the BS. By contrast, during the third phase seen in Fig. 2c, RS2 forwards the BS's DL signal $\mathbf{c}_{2}$ to the MS, while RS1 listens to the next DL signal $\mathbf{c}_{3}$ received from the BS. In this manner the two relays alternatively transmit and listen. In this scenario we could argue that both the direct and relayed copies of $\mathbf{c}_{1}, \mathbf{c}_{2}$, and $\mathbf{c}_{3}$ were received and hence secondorder diversity is attained, which is achieved at the cost of invoking two RSs. Hence, in this relaying scheme $(N+1)$ communication phases are required to convey $N$ packets with the aid of two relays, so second-order diversity is achieved while maintaining almost the same slot efficiency as direct communication operating without relaying, provided that $N$ is sufficiently high.

As seen in Fig. 2c, in the successive relaying scheme, the received signal of RS1 is subjected to the interfering signal of RS2 during phase 3. Similarly, observe in Fig. $2 b$ that during phase 2 the reception of RS2 is contaminated by the

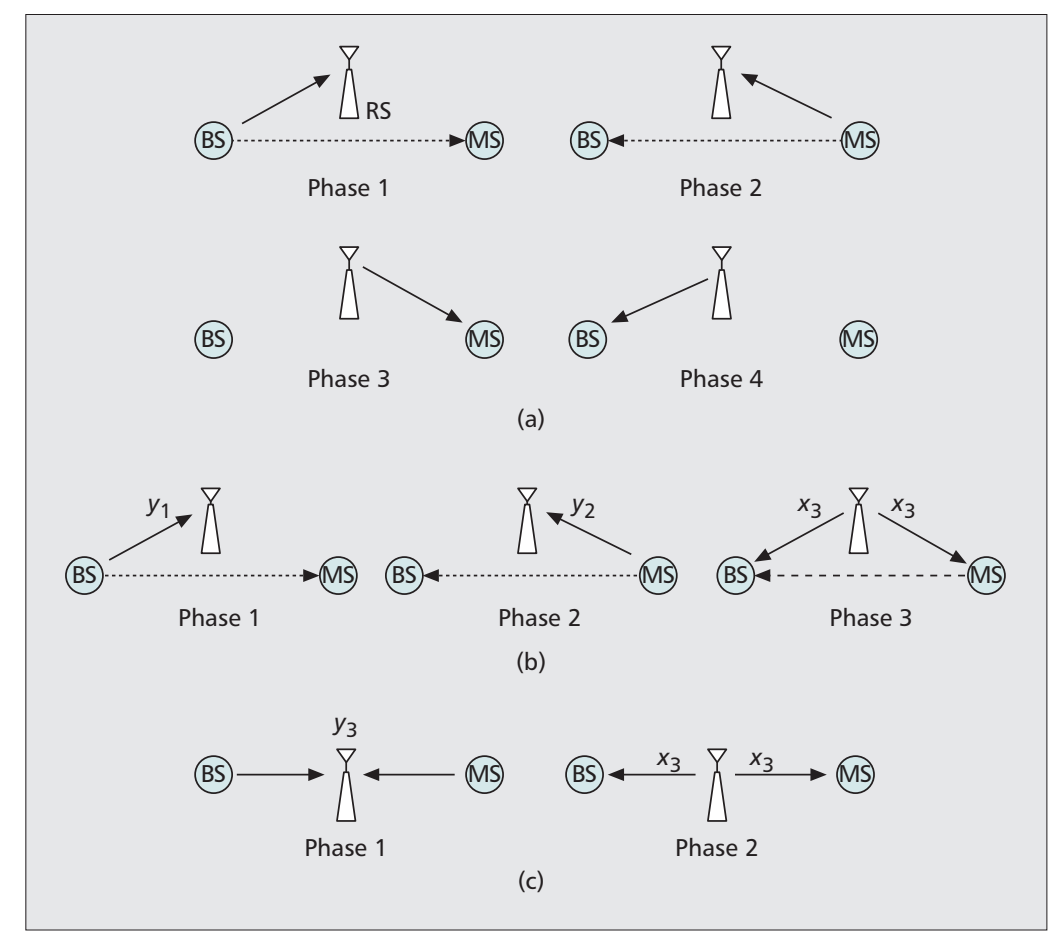

Figure 1. Relaying protocols: a) traditional four-phase relaying; $b$ ) three-phase relaying; c) two-phase relaying.

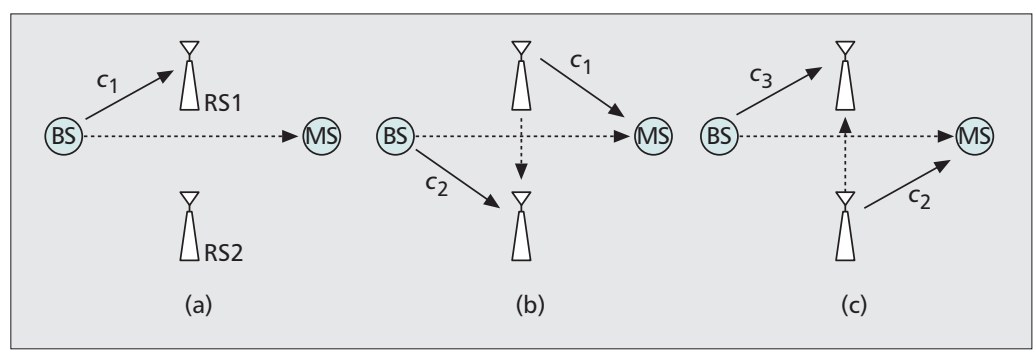

Figure 2. Successive relaying protocol: a) phase 1; b) phase 2; c) phase 3.

transmission of RS1. It is typically assumed that either this interfering signal has sufficiently low power or, alternatively, sufficiently high power that its effect can be cancelled from the desired signal. However, in realistic environments the signal power received at the other relay fluctuates, depending on the channel conditions between the two relays. Therefore, the RS in receive mode typically suffers from the interference inflicted by the other RS, and this is likely to degrade the overall system performance. Furthermore, the successive relaying protocol of Fig. 2 requires two RSs, while traditional relaying requires only a single RS. This implies that more RSs are required to support successive relaying, and this potentially increases the overall infrastructure cost, unless the RSs are constituted by inactive MSs.

\section{Network Coding Aided THREE-PhASE DF RELAYING}

In contrast to the successive relaying protocol, the network coding (NC) aided relaying protocol [5-7] is capable of operating without requiring 
To further reduce

the required

communication

resources, three

different types of

two-phase relaying

protocols may be

invoked: two-phase

AF, denoise-and-

forward (DNF), and

DF protocols.

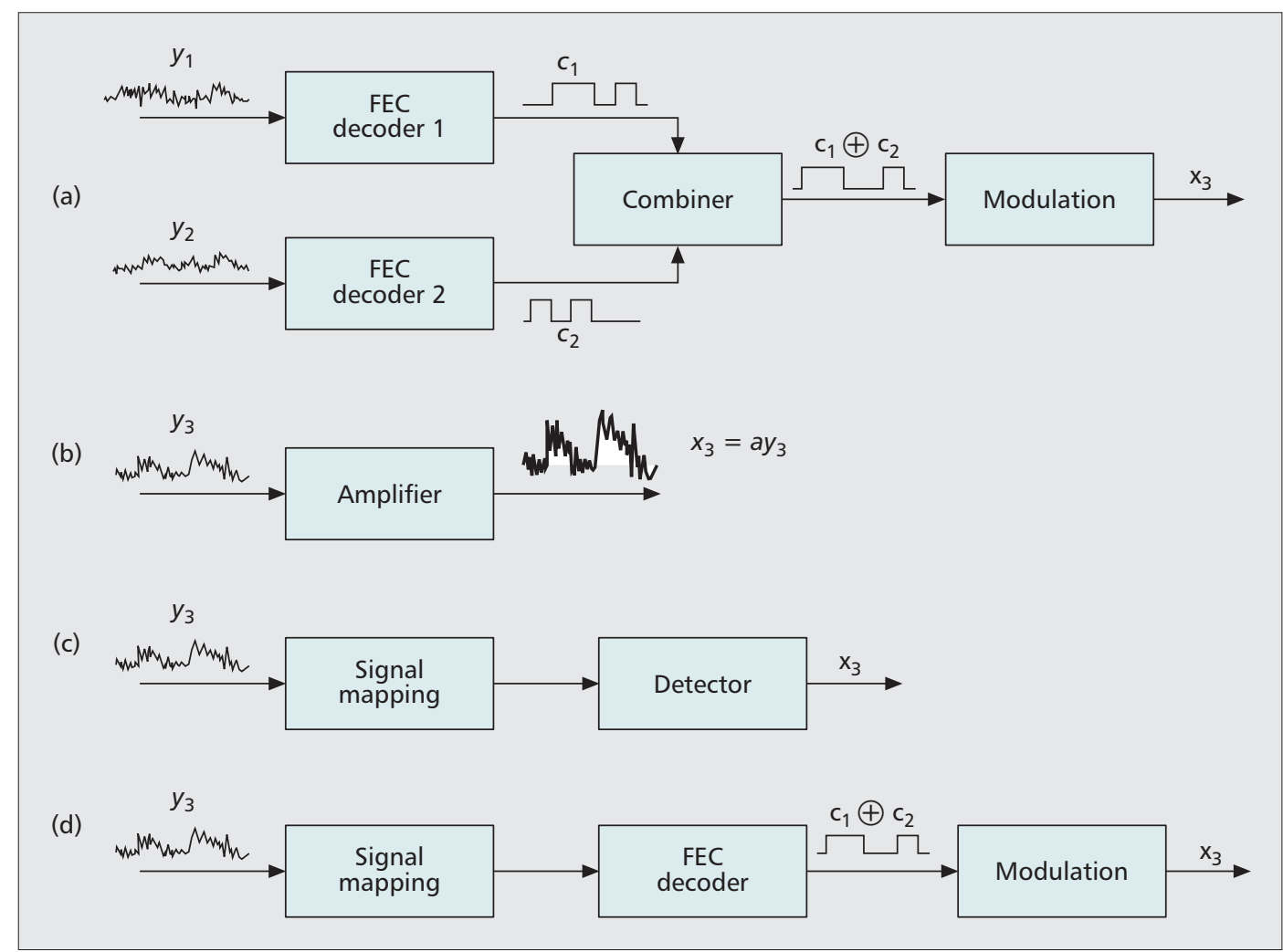

Figure 3. Block diagrams of three-phase and two-phase relaying protocols: a) three-phase DF; $b$ ) two-phase $A F$; ) two-phase DNF; d) two-phase DF.

any additional RS. Figure $1 \mathrm{~b}$ depicts the basic philosophy of the NC-aided three-phase relaying system, and the relay's operation is further detailed in Fig. 3a. The BS and MS transmit the codewords $\mathbf{c}_{1}$ and $\mathbf{c}_{2}$ during the first and second communication phases to the RS, respectively. Then the RS independently decodes the corresponding received signals $\mathbf{y}_{1}$ as well as $\mathbf{y}_{2}$ and combines the decoded codewords into a single stream. More explicitly, the relay performs the bit-wise exclusive OR (XOR) operation of the two packets to obtain the resultant composite packet $\mathbf{c}_{3}=\mathbf{c}_{1} \oplus \mathbf{c}_{2}$. This operation may be interpreted as informing both destinations of the bit positions of the transmission packet, where the remote transmitter's bits were different from those of the local transmitter, as indicated by their XOR function. If two packets have different lengths, either zero padding or repetition coding can be applied to the shorter one in order to make their lengths identical.

As seen in Fig. 1b, in the third phase the composite network-coded packet is modulated and broadcast to both the BS as well as to the MS. The signal received at each destination can be regarded as the combination of the UL and DL packets. To recover the desired packet at each destination node, each node utilizes the a priori knowledge of its own transmitted packet that was transmitted in the previous communication phase. For example, the MS first computes the log-likelihood ratios (LLRs) of the networkcoded bits. To extract the soft information related to the DL packet, we flip the sign of the network-coded bit's LLR if the corresponding UL bit was a logical 1 based on the XOR func- tion, while maintaining its magnitude. As in the traditional four-phase regime of Fig. 1a, the signal directly received from the source can also be exploited with the aid of soft or hard packet combining in order to attain diversity gain.

The three-phase DF relaying scheme of Fig. $1 \mathrm{~b}$ requires only three time slots to complete a full cycle of UL and DL transmissions. Therefore, we can expect a 33 percent throughput increase compared to the traditional relaying regime of Fig. 1a, which requires four communication phases. It is worth noting that the NCaided three-phase DF relaying protocol can readily be applied to arbitrary high-order modulation schemes, such as 16-quadrature amplitude modulation (QAM), 64-QAM, and multipleinput multiple-output (MIMO) systems.

\section{TWO-PhASe RELAYING}

To further reduce the required communication resources, three different types of two-phase relaying protocols may be invoked: two-phase $\mathrm{AF}$, denoise-and-forward (DNF), and DF protocols. The two-phase relaying protocols of Fig. 1c are often referred to as two-way relaying or bidirectional relaying, as opposed to the traditional four-phase relaying protocol, which is also often referred to as one-way relaying. The relay's actions in various two-phase relaying protocols are illustrated in Fig. 3.

\section{TWO-PHASE AF RELAYING}

In two-phase AF relaying [4, 8], the MS and BS simultaneously transmit their signals to the RS during the first phase, as shown in Fig. 1c; hence 
the RS receives the superposition of these two signals, which is denoted $\mathbf{y}_{3}$. In the second phase the RS amplifies the composite received signal while obeying a specific total power constraint, and forwards it to both the MS and BS. We assume that the BS receives the sufficiently accurately quantized channel impulse responses (CIRs) $h_{M R}$ of the MS-RS link and $h_{R B}$ of the RS-BS link. Exploiting the knowledge of the CIRs and of its own transmitted data, the MS may be able to perfectly cancel the effects of the superimposed UL signal from its received signal. Accordingly, the MS is capable of decoding the desired DL packet. In a similar manner the BS performs decoding of the UL packet. Even though the two-phase AF relaying regime of Fig. $3 \mathrm{~b}$ does not require additional communication phases compared to the classic direct communication, its performance is affected by the inevitable noise amplification. Furthermore, the RS simultaneously conveys both the UL and DL signals, which typically requires increased power. More explicitly, the MS only needs the DL signal; however, the signal transmitted from the RS to the MS also contains the UL signal, which increases the required power. By contrast, the NC-aided three-phase DF relaying scheme of Fig. 1b does not require any additional power, since it transmits the XOR-ed packet from the RS rather than the sum of the UL and DL signals. Hence, the two-phase AF relaying protocol typically results in an SNR performance loss in comparison to three-phase relaying, even though it reduces the number of time slots required, which contradicts our original objective of increasing the achievable SNR.

\section{Network Coding Aided Two-Phase Relaying}

To reduce the effect of noise amplification in the two-phase AF regime, the DNF relaying protocol, which is based on the above-mentioned network coding scheme, has been proposed in $[9,10]$. As in the AF two-phase relaying regime of Fig. 3b, the RS of Fig. 3c receives both the UL and DL signals in the first phase. The denoising operation may also be referred to as detect and forward, because it is constituted by the noise elimination process of the detector's slicing or decision operation. Then the RS of Fig. 3c detects the symbols by processing the received signal and attempts to estimate the XOR-based composite symbol values $\mathbf{x}_{3}$, rather than separately detecting the UL and DL symbols of $\mathbf{x}_{1}=$ $Q\left(\mathbf{c}_{1}\right)$ and $\mathbf{x}_{2}=Q\left(\mathbf{c}_{2}\right)$, where $Q(\cdot)$ denotes the modulation of coded bits. For example, if we assume that both the BS-RS CIR $h_{B R}$ as well as the MS-RS CIR $h_{M R}$ have a value of unity and that quaternary phase shift keying (QPSK) modulation is used, the I and Q component of the RS's received signal in each time slot has the values of $\{-2,0,2\}$ in the absence of noise. Here, the power normalization of QPSK signaling is ignored for simplicity. Then, by using the mapping function of $f(-2)=1, f(0)=-1, f(2)=1$, we obtain the combined signal of the UL and DL packets. More specifically, for QPSK signaling this mapping function directly generates the modulated signal stream $\mathbf{x}_{3}$ of Fig. $3 \mathrm{c}$ associated with the XOR-ed function of the UL and DL packets, which is the same as the transmit signal of the three-phase DF RS seen in Fig. 3a and described earlier. Similar to the three-phase DF relaying of Fig. 1b, each destination node is capable of extracting its own desired packet from the composite received signal by exploiting the a priori knowledge of its transmitted symbol stream. It was shown in [9] that the symbol-wise mapping function of $f(\cdot)$ exists and can be constructed for arbitrary regular $M$-QAM modulation schemes.

In contrast to the two-phase AF relaying of Fig. 3b, the noise amplification problem does not occur in the DNF relaying protocol of Fig. $3 c$, since the denoised (i.e., detected or sliced) symbol stream is transmitted from the RS with the aid of the specific mapping function $f(\cdot)$ exemplified above for QPSK. However, the RS of the DNF scheme does not take advantage of channel coding and hence may have a high error probability for the relayed signal.

In contrast to the DNF regime of Fig. 3c, which dispenses with FEC coding, in the twophase DF relaying of Fig. 3d, the RS performs FEC decoding in order to mitigate the effects of error propagation. A possible approach $[4,11]$ not depicted here for the two-phase DF relaying protocol is that the RS separately decodes the codewords $\mathbf{c}_{1}$ and $\mathbf{c}_{2}$, which are transmitted from the MS and BS, respectively. The RS receives the superposition of the UL and DL signals in the first phase and attempts to decode both of them. During the second phase of Fig. 1c, the $\mathrm{RS}$ generates the composite packet, which is broadcast to both the MS and BS, as in the three-phase DF relaying scheme of Fig. 1b. In the second communication phase, each destination node decodes its desired signal in the same way as the three-phase relaying regime of Fig. 1b. Nonetheless, its decoding performance at the RS is typically worse than that of the threephase DF relaying protocol, where only one of the UL and DL packets arrives and is decoded at the RS in each phase.

For comparison, in another two-phase DF relaying approach $[10,12]$ of Fig. $3 d$, it is assumed that the UL and DL packets are encoded by identical linear FEC encoders having identical coding rates and code lengths. As in the three-phase DF relaying of Fig. 3b, both zeropadding and repetition coding can be used to render the UL and DL packets length identical. We also assume that the channel is pre-equalized so that the effective CIRs $h_{M R}$ and $h_{B R}$ have a value of unity as in the DNF relaying of Fig. 3c. Under this assumption, the superposition of two binary codewords can readily be mapped to a valid codeword according to $\mathbf{c}_{3}=\mathbf{c}_{1} \oplus \mathbf{c}_{2}$ by exploiting the specific property of linear codes that the linear combination of two codewords constructed over the Galois field GF(2) also becomes a valid codeword. For QPSK signaling, the superposition of the UL and DL symbols has a value of $\{-2,0,2\}$ for both the real and imaginary components. Similar to the DNF relaying of Fig. 3c, the mapping function of $g(-2)=0, g(0)$ $=1, g(2)=0$ is utilized to transform the superposition of two codewords into the addition operation over the Galois field. We note that $g(\cdot)$ generates the binary composite coded bits $\mathbf{c}_{3}$, while the mapping function $f(\cdot)$ of DNF relay-
The two-phase AF relaying protocol typically results in an SNR performance loss in comparison to three-phase relaying, even though it reduces the number of time slots required, which contradicts to our original objective of increasing the achievable SNR. 


\begin{tabular}{|llllllll}
\hline Relaying protocol & 4-AF & 4-DF & Successive relaying & 3-DF & 2-AF & 2-DNF & 2-DF \\
\hline Spectral efficiency & Low & Low & High & Medium & High & High & High \\
\hline Effective SINR & Low & High & Medium & High & Very low & Medium & High \\
\hline System constraint & Low & Low & High & Medium & Low & Medium & Very High \\
\hline Number of relays & 1 & 1 & 2 & 1 & 1 & 1 & 1 \\
\hline Complexity & Low & High & High & High & Low & Medium & High \\
\hline Schematic figure & 1 a & 1 a & 2 & 1b, 3a & 1c,3b & 1c,3c & $1 c, 3 d$ \\
\hline
\end{tabular}

Table 1. Comparison of relaying protocols.

\begin{tabular}{|llll|}
\hline Relaying schemes & 4-phase & 3-phase & 2-phase \\
\hline Number channel uses per phase & 512 & 512 & 512 \\
\hline Modulation & $16-\mathrm{QAM}$ & $16-\mathrm{Q} A \mathrm{M}$ & QPSK \\
\hline Code rate & $1 / 2$ & $3 / 8$ & $1 / 2$ \\
\hline Code length & 2048 & 2048 & 1024 \\
\hline Number of information bits per codeword & 1024 & 768 & 512 \\
\hline No. of information bits (UL + DL) per phase & 512 & 512 & 512 \\
\hline No. of information bits per channel use & 1 & 1 & 1 \\
\hline Relative power of each transmission & 1 & 1 & $2 / 3$ \\
\hline
\end{tabular}

Table 2. Simulation parameters. munication regime. Therefore, we can expect that the two-phase DF regime of Fig. 3d provides both high slot efficiency as well as high BER performance. Nonetheless, only the mapping algorithms of BPSK and QPSK modulation are known at the time of writing; hence, the appreciation of the two-phase DF relaying of Fig. 3d to higher-order modulation schemes such as 16-QAM and 64-QAM constitutes an open problem. Furthermore, identical FEC codes having the same code length in the UL and DL must be used, and this constraint reduces the flexibility of the system.

In Table 1 the characteristics of the aforementioned relaying schemes of Figs. 1-3 are summarized.

\section{Numerical Results}

In this section the achievable performance of the relaying protocols considered is compared. The SNR is defined as the ratio between the signal power and the noise variance. It is assumed that 512 QPSK-modulated symbols per packet are transmitted during a communication phase, and turbo codes having various rates and interleaver lengths are employed. The overall rate (the number of DL and UL information bits per channel use) is set to unity for all the relaying schemes. The detailed parameters are presented in Table 2. We note that for the two-phase AF, DNF and DF relaying protocols of Fig. 1c, a 1/3 lower transmit power per transmission phase is allocated compared to that of the three-phase and four-phase relaying protocols for the sake of fair comparison, because the MS and BS simultaneously transmit their signals during the first phase. By allocating the relative power of $2 / 3$ to each of the MS, BS, and RS, the transmit power allocated in the first and second phases becomes $4 / 3$ and $2 / 3$, respectively; hence, the average transmit power per transmission phase is unity.

Figure 4a illustrates the packet error rate (PER) performance of the UL in the relaying systems of Figs. 1 and 3. The SNR $\gamma_{M R}$ between the MS and the RS is assumed to be the same as the SNR $\gamma_{B R}$ between the BS and the RS, which implies that the RS is approximately halfway between the BS and MS. The SNR of the direct link from the MS to the BS is denoted $\gamma_{M B}$. We also assume that the relay channel has a higher SNR than the direct channel (i.e., $\gamma_{M R} \geq \gamma_{M B}$ ), 


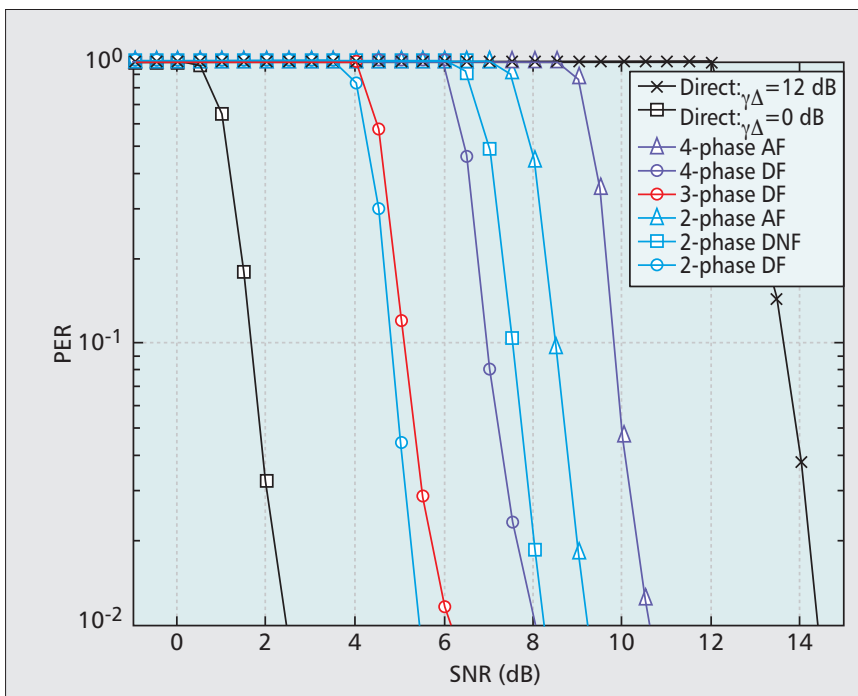

(a)

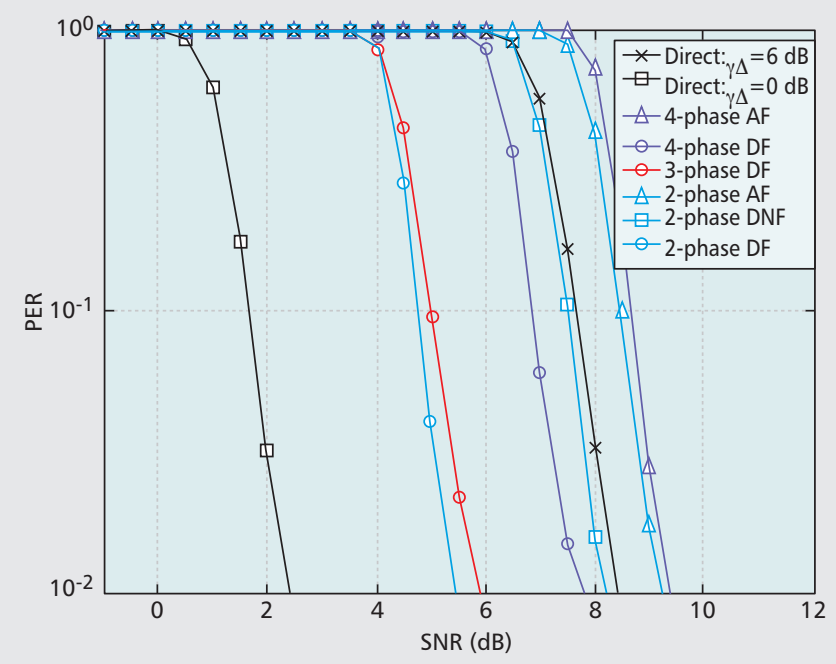

(b)

Figure 4. PER vs. SNR performance: a) $\left.\gamma_{\Delta}=12 d B, ; b\right) \gamma_{\Delta}=6 d B$. The two-phase and three-phase DF protocols of Fig. $3 a$ and $3 d$, which employ network coding, outperform the other relaying protocols.

and the difference between these two SNRs is set to $\gamma \Delta=12 \mathrm{~dB}$. As a performance bound, the direct communication scenario associated with $\gamma \Delta=0 \mathrm{~dB}$ is also considered, which corresponds to the scenario in which the SNR of the direct link is as high as that of the relay link. Successive relaying was not investigated, since its performance is highly affected by the interference between the two RSs. However, if the absence of inter-relay interference is assumed and the SNR of the relay link is significantly higher than that of the direct link, the performance of successive relaying is approximately $3 \mathrm{~dB}$ worse than that of the direct link associated with $\gamma \Delta=0 \mathrm{~dB}$, which implies that it only has a $3 \mathrm{~dB}$ power penalty due to the simultaneous transmissions from the RS and the source nodes.

Observe in Fig. 4a that the two-phase DF relaying protocol of Fig. 1d has the best PER performance among all the relaying schemes considered, because it employs a lower-order modulation scheme than the three-phase and four-phase relaying protocols at the same overall transmission rate. We also note that here the two-phase DF relaying protocol decodes the composite packet at the RS, rather than separately decoding the DL and UL packets. The BER curve of the three-phase DF regime of Fig. $3 \mathrm{a}$ is only $0.6 \mathrm{~dB}$ away from the two-phase $\mathrm{DF}$ relaying of Fig. 3d, both of which employ the network coding scheme. Figure 4a also demonstrates that the two-phase DNF and AF schemes of Fig. 3a and $3 b$ are outperformed by the fourphase DF regime of Fig. 1a, which implies that the error propagation and noise amplification effects erode their advantage of higher slot efficiency.

Figure $4 \mathrm{~b}$ characterizes the PER performance of the various relaying protocols for $\gamma \Delta=6 \mathrm{~dB}$. It is seen that the performance of the threephase DF relaying of Fig. 3a is slightly improved as a benefit of cooperative reception. Interestingly, the direct communication regime having $\gamma \Delta=6 \mathrm{~dB}$ shows approximately $1 \mathrm{~dB}$ SNR gain compared to the two-phase and four-phase AF protocols of Figs. 1a and 3b. The noise amplification and reduced low slot efficiency erode the benefits of the four-phase AF relaying of Fig. 1a, when the SNR difference between the relay link and the direct link is small. In the two-phase AF relaying regime of Fig. $3 \mathrm{~b}$, the SNR loss due to the simultaneous transmission of the UL and DL signals from the RS also degrades its end-to-end performance.

\section{CONCLUSION}

In this overview we have described the various wireless relaying protocols summarized in Table 1. The traditional relaying protocol of Fig. 1a is capable of improving the achievable end-to-end channel quality, but it requires four communication phases (i.e., time slots), which is twice as many as in classic direct communication. The successive relaying scheme requires nearly the same number of time slots as direct communication, but it needs an additional relay. As another approach to save valuable communication resources, network coding may be adopted in the three-phase DF relaying of Figs. $1 \mathrm{~b}$ and $3 \mathrm{a}$. The two-phase AF and DNF relaying schemes of Fig. $3 \mathrm{~b}$ and $3 \mathrm{c}$ require only two phases for a pair of UL and DL transmissions, although their performances are degraded by noise amplification and error propagation. The two-phase DF relaying of Fig. 3d is also based on network coding and it provides good PER performance as well as high slot efficiency. Nonetheless, it imposes more rigid system constraints than the other relaying protocols. To implement the abovementioned relaying protocols in future wireless systems, a number of key problems should be resolved.

The numerical results of Fig. 4 show that the three-phase and two-phase DF relaying schemes of Fig. 3a and 3d, which are both based on network coding, outperform the other relaying schemes. However, when adopting network coding, joint design of the DL and UL signals is 
It is worth studying the efficient

estimation, quantization and transmission of the CIR information in relaying systems. Furthermore, non-coherent relaying algorithms, which do not require channel information, have to be studied. required, which imposes additional constraints on the overall system. More specifically, in the two-phase DF relaying scheme of Fig. 3d, the design of attractive power control and channel assignment strategies substantially affects the decoding performance. When network coding is employed in practical systems, the combined DL/UL scheduling algorithm, which can jointly control the modulation and coding scheme of the DL and UL, is also required in order to maximize the system's throughput. Furthermore, efficient mapping algorithms designed for highorder 16-QAM and 64-QAM should be developed in order to improve the practicability of the two-phase DF relaying protocol of Fig. 3d.

To increase the attainable slot efficiency, the employment of MIMO appears promising, especially in the context of MIMO-aided transmit preprocessing. In the three-phase and two-phase relaying protocols, the UL and DL signals are simultaneously transmitted, and hence the transmit preprocessor should be designed to jointly optimize both the UL and DL transmissions. Furthermore, using a space-division multiple access (SDMA)-aided relaying scheme also constitutes a possible way of relaying signals. By exploiting spatial signal processing, the SDMAaided RS equipped with multiple antennas is capable of receiving or transmitting the DL and UL packets at the same time. Combining SDMAaided relaying with network coding constitutes a promising research topic, with the potential to further improve the achievable performance of spectrally efficient relaying protocols.

In practical RS-aided systems, the destination node should estimate both the relay channel as well as the direct channel. In AF relaying the channel information between the source and the relay is also required at the destination, which implies a high burden on the overall system, especially when the signal is relayed via more than two hops. To resolve this problem, it is worth studying the efficient estimation, quantization, and transmission of the CIR information in relaying systems. Furthermore, non-coherent relaying algorithms, which do not require channel information, have to be studied.

\section{REFERENCES}

[1] R. Pabst et al., "Relay-Based Deployment Concepts for Wireless and Mobile Broadband Radio," IEEE Commun. Mag. vol. 42, no. 9, Sept. 2004, pp. 80-89.

[2] D. Soldani and S. Dixit, "Wireless Relays for Broadband Access," IEEE Commun. Mag., vol. 46, no. 3, 2008, pp. 58-66.

[3] Y. Fan et al., "Recovering Multiplexing Loss through Successive Relaying Using Repetition Coding," IEEE Trans. Wireless Commun., vol. 6, no. 12, Dec. 2007, pp. 4484-93.

[4] B. Rankov and A. Wittneben, "Spectral Efficient Protocols for Half-Duplex Fading Relay Channels," IEEE JSAC vol. 25, no. 2, Feb. 2007, pp. 379-89.
[5] Y. Wu, P. A. Chou, and S.-Y. Kung, "Information Exchange in Wireless Networks with Network Coding and Physical-Layer Broadcast," Proc. 39th Annual Conf. Info. Sci. Sys., Mar. 2005.

[6] P. Larsson, N. Johansson, and K.-E. Sunell, "Coded Bidirectional Relaying," Proc. IEEE VTC, vol. 2, 2006, pp. $851-55$

[7] K. Lee and L. Hanzo "Multiple Antenna Assisted Hard versus Soft Decoding-and-Forwarding for Network Coding Aided Relaying Systems," Proc. IEEE GLOBECOM '08, Nov. 2008, pp. 1-5.

[8] P. Popovski and H. Yomo, "Bidirectional Amplification of Throughput in a Wireless Multi-Hop Network," Proc. IEEE VTC, vol. 2, May 2006, pp. 588-93.

[9] S. Zhang, S. Liew, and P. Lam, "Physical-Layer Network Coding," Proc. 12th ACM MobiCom, 2006, pp. 358-65

[10] P. Popovski and H. Yomo, "The Anti-Packets can Increase the Achievable Throughput of a Wireless Multi-Hop Network," Proc. IEEE ICC, vol. 9, June 2006 , pp. 3885-90.

[11] S. J. Kim, P. Mitran, and V. Tarokh, "Performance Bounds for Bi-directional Coded Cooperation Protocols," Proc. ICDCSW '07, June 22-29, 2007.

[12] H. J. Yang, K. Lee, and J. Chun, "Zero-forcing Based Two-phase Relaying," Proc. IEEE ICC, June 2007, pp. 5224-28.

\section{AdDitional ReAding}

[1] T. Unger and A. Klein, "On the Performance of Two-way Relaying with Multiple-Antenna Relay Stations," Proc. 16th IST Mobile Wireless Commun. Summit, July 2007, pp. 1-5.

\section{BIOGRAPHIES}

KYUNGCHEN LEE (kl7v@ecs.soton.ac.uk) received B.S., M.S. and $\mathrm{Ph} . \mathrm{D}$. degrees in electrical engineering from Korea Advanced Institute of Science and Technology (KAIST), Daejeon, in 2000, 2002, and 2007, respectively. From September 2005 until February 2006 he was a visiting research student with the Communication Research Group, University of Southampton, United Kingdom, where he held a postdoctorial visiting research fellowship from April 2007 until June 2008. Currently, he is with Samsung Electronics, Suwon, Korea. His research interests include signal processing and optimization for wireless communications with focus on MIMO, cooperative systems, and cross-layer design.

LAJOS HANZO [F] (Ih@ecs.soton.ac.uk), FREng, FIET, received his degree in electronics in 1976, his doctorate in 1983 and his D.Sc. degree in 2004. During his 34-year career in telecommunications he has held various research and academic posts in Hungary, Germany, and the United Kingdom. Since 1986 he has been with the School of Electronics and Computer Science, University of Southampton, where he holds the chair in telecommunications. He has coauthored 19 Wiley-IEEE Press books on mobile radio communications totaling in excess of 10,000 pages, published 684 research papers at IEEE Xplore, acted as TPC Chair of IEEE conferences, presented keynote lectures, and been awarded a number of distinctions. Currently he is directing an academic research team working on a range of research projects in the field of wireless multimedia communications sponsored by industry, the Engineering and Physical Sciences Research Council (EPSRC) United Kingdom, the European IST Program, and the Mobile Virtual Centre of Excellence (VCE), United Kgindom. He is an enthusiastic supporter of industrial and academic liaison, and he offers a range of industrial courses. He is also an IEEE Distinguished Lecturer as well as a Governor of both the IEEE Communications Society and the VTS. He is the acting Editor-in-Chief of IEEE Press. For further information on research in progress and associated publications please refer to http://www-mobile.ecs.soton.ac.uk. 